\title{
Near infrared photometry of comet C/2005 E2 (McNaught)
}

\section{Enos Picazzio $^{1}$, Elysandra Figueredo ${ }^{1}$, Amaury Augusto de Almeida ${ }^{1}$, Claudia Mendes de Oliveira ${ }^{1}$, and Klim Ivanovich Churyumov ${ }^{2}$}

${ }^{1}$ Department of Astronomy, Institute of Astronomy, Geophysics and Atmospheric Sciences, University of São Paulo,

Rua do Matão 1226, Cidade Universitária 05508-900 São Paulo SP, BRAZIL

email:picazzio@astro.iag.usp.br

${ }^{2}$ Astronomical Observatory, Kyiv Shevchenko National University,

Box 04053, Observatorna str., 3, Kyiv, Ukraine

email: klim.churyumov@observ.univ.kiev.ua

\begin{abstract}
We present results of JHK photometry for comet C/2005 E2 (McNaught). Observations were made with the OSIRIS imager at the SOAR telescope, on Sept. 26-27, 2005. The dependence of the main parameters on the angular distance from the cometary photometric nucleus is discussed. We considered concentric rings around the photometric nucleus of the comet, with the radius determined by the aperture. Integrated flux in the ring, surface brightness, J-H and H-K color indices, and mean flux decay were obtained for each ring. A phometric radial profile was also obtained by tracing average values of the ace brightness for increasing values of radii in isophotes which allowed us to compute the azimuthally averaged surface brightness of the coma. The images centered on the photometric nucleus show three jets from active areas on the nucleus.
\end{abstract}

Keywords. comets: general, comets: individual C/2005 E2 (McNaught), techniques: image processing

\section{Observations and processing}

Comet C/2005 E2 (McNaught) was discovered on Mar. 12, 2005, when it was 15.5 mag., with a uniform 7 arcsec coma and 13 arcsec tail in phase angle $250^{\circ}$. It is a non-periodic comet (eccentricity $=1.0001388$ ), with an orbital plane inclination of $17^{\circ}$, perihelion distance $=1.5196 \mathrm{AU}$, and perihelion on Feb. 23.4729229, 2006 .

We observed C/2005 E2 on Sept. 26-27, 2005, at a geocentric distance of $\Delta=2,492$ $\mathrm{AU}$, a heliocentric distance of $r=2.050 \mathrm{AU}$, and a phase angle of $22.9^{\circ}$, with a magnitude $m_{V}=11.5$. Images were taken with OSIRIS (Ohio State InfraRed Imager/Spectrometer, $\mathrm{F} / 7, \mathrm{FOV}=80 \mathrm{arcsec}$, and plate scale $=0.139 \mathrm{arcsec} / \mathrm{pixel}$ ) at the $4.1-\mathrm{m} \mathrm{SOAR}$ (Southern Astrophysical Research telescope, Cerro Pachón, Chile). FWHM in arcsec of individual images in the wavebands are: $0.88(\mathrm{~J}), 0.84(\mathrm{H})$ and $0.83(\mathrm{~K})$. All images were corrected in terms of OSIRIS linearity coefficients and flatfield. Bad pixel mask and sky subtraction were accomplished using IRAF and Cirred (CTIO IR Reduction Package). The calibration star is P9181 (S234-E) from Persson et al. (1998), with the following magnitudes: $\mathrm{J}=12.464 \pm 0.011, \mathrm{H}=12.127 \pm 0.008$, and $\mathrm{K}=12.095 \pm 0.007$. 


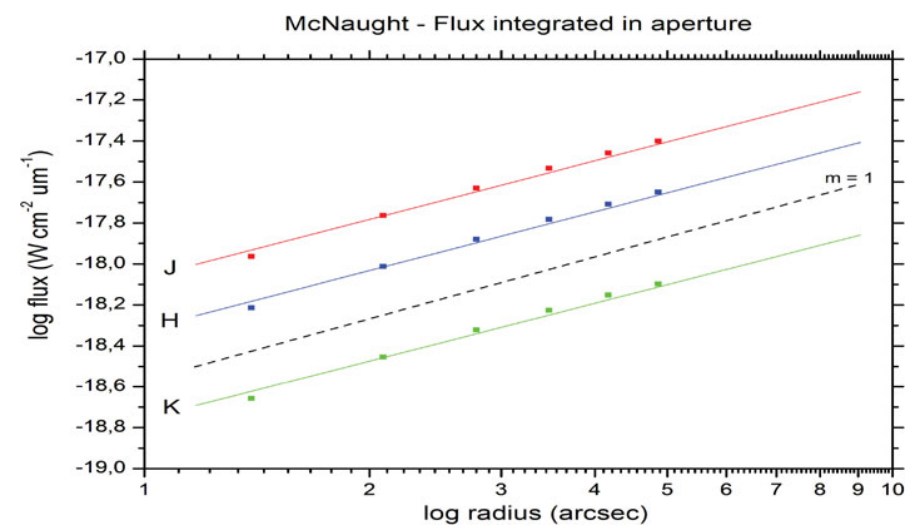

Figure 1. Surface brightness profile of Comet C/2005 E2 (McNaught). Also, plotted for reference is the case $m=1$ line for steady state model.

\section{Discussion}

\subsection{Surface brightness profile}

Standard models invoking spherically symmetric constant dust production and optically thin coma predict that the surface brightness will fall with nucleocentric distance as $r^{-1}$ [Gehrz \& Ney (1992)]. Integrating the surface brightness over a circular aperture of the angular radius centred at the comet nucleus we obtain the brightness with a logarithmic slope $m$ ( $m=1$ for a steady-state isotropic model and $m=1.5$ for a radiation pressure dominated case).

As it can be seen from Fig. 1, the surface brightness of comet McNaught falls approximately as $r^{-1}$. For apertures smaller than 1 arcsec the surface brightness falls steeper, but there the fitting is not good.

\subsection{Integrated magnitude}

The dependence of the surface brightness on the aperture for comets McNaught and $1 \mathrm{P} /$ Halley is shown in Fig. 2. Comet McNaught presents similar behaviour in the $\mathrm{J}, \mathrm{H}$ and $\mathrm{K}$ bands, but for comet Halley the similarity on $\mathrm{J}$ and $\mathrm{K}$ bands appears only on March 10 [Woodward et al. (1996)]. The surface brightness varies more steeply for comet Halley.

The magnitudes integrated in $10 \operatorname{arcsec}(m)$ and the absolute magnitudes $\left(H_{0}=m-\right.$ $5 \log \Delta-2.5 n \log r)$ in each waveband are: $\mathrm{J}\left(m=11.75, H_{0}=6.33\right), \mathrm{H}(m=11.29$, $\left.H_{0}=5.84\right)$ and $\mathrm{K}\left(m=11.28, H_{0}=5.74\right)$. The photometric index $(n)$ for the preperihelion phase was taken from Yoshida (2008).

The color of comet McNaught seems to be bluer than the Sun (Fig.3). It is known that fine grains scatter blue light much better than red light. Comet Halley, for example, showed fine dust grains near the nucleus.

\subsection{Color indices}

Color indices for comets McNaught and Halley, and Kuiper Belt Objects (KBOs) are shown in Table 1. Comet McNaught is very similar to some KBOs (Classical, Scattered and Resonant).

\subsection{Morphology of comet McNaught (C/2005 E2)}

Broadband images in J, H and $\mathrm{K}$ are shown in Fig.5. Three jets are projected on the plane of the sky. They seem to be very similar in the different bands. The $\mathrm{K}$ band image 


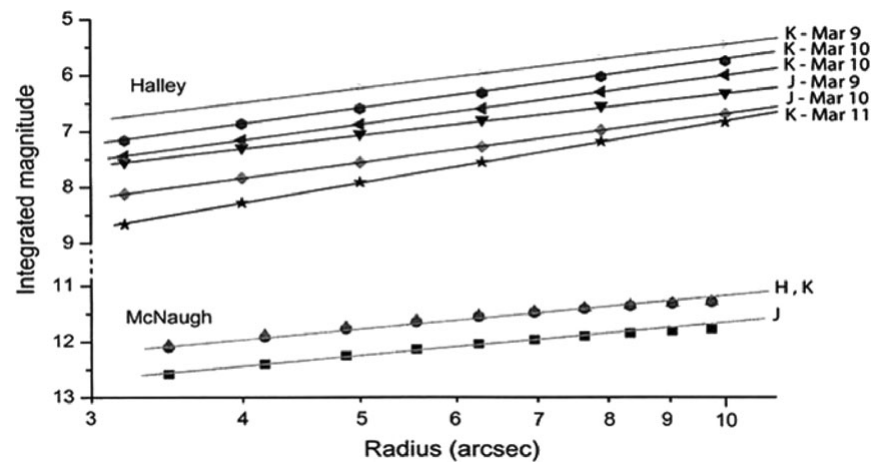

Figure 2. Integrated magnitude for comets 1P/Halley and C/2005 E2 (McNaught).

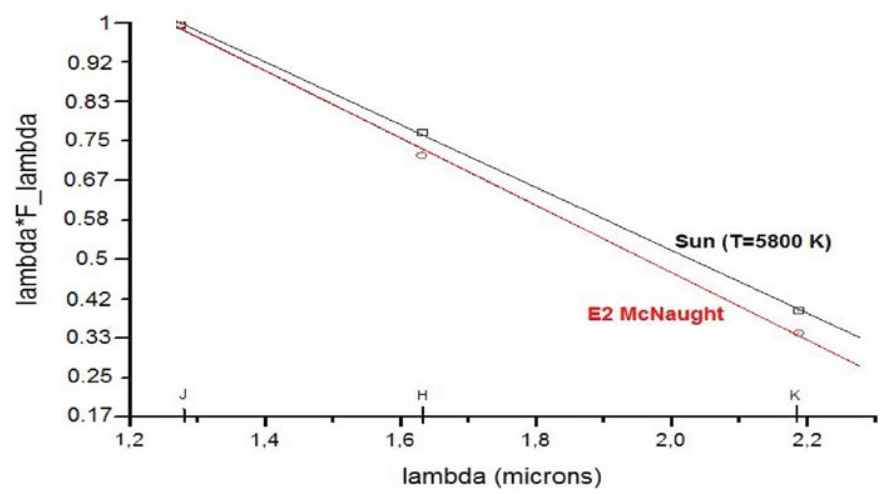

Figure 3. Color of comet McNaught and for the Sun.

Table 1. Color index for comets $\mathrm{C} / 2005 \mathrm{E} 2$ and 1P, and for KBOs.

\begin{tabular}{lccccc}
\hline Object & $\mathrm{J}-\mathrm{H}$ & $\mathrm{H}-\mathrm{K}$ & $\mathrm{J}-\mathrm{K}$ & Nature & Ref \\
\hline McNaugth & $0.48 \pm 0.01$ & $0.01 \pm 0.01$ & $0.49 \pm 0.01$ & Oort Cloud & $\mathrm{A}$ \\
Halley & $0.48 \pm 0.02$ & $0.17 \pm 0.03$ & $0.65 \pm 0.05$ & Jupiter's family & $\mathrm{B}$ \\
Halley & 0.69 & 0.25 & 0.94 & Jupiter's family & $\mathrm{G}$ \\
$1999 \mathrm{CD}_{158}$ & $0.44 \pm 0.10$ & $0.03 \pm 0.10$ & $0.47 \pm 0.09$ & Classical KBO & $\mathrm{C}, \mathrm{D}$ \\
$2000 \mathrm{OK}_{67}$ & $0.46 \pm 0.08$ & $0.04 \pm 0.07$ & $0.50 \pm 0.09)$ & Classical KBO & $\mathrm{C}$ \\
$1996 \mathrm{GQ}_{21}$ & $0.48 \pm 0.07$ & $0.05 \pm 0.08$ & $0.68 \pm 0.11$ & KBO-SDO* & $\mathrm{D}, \mathrm{E}, \mathrm{F}$ \\
$1998 \mathrm{VG}_{44}$ & $0.41 \pm 0.06$ & $0.01 \pm 0.08$ & $0.42 \pm 0.08$ & Resonant KBO & $\mathrm{E}$ \\
$1999 \mathrm{RZ}_{253}$ & $0.48 \pm 0.09$ & $0.10 \pm 0.09$ & $0.58 \pm 0.06$ & Classical KBO & $\mathrm{E}, \mathrm{F}$ \\
\hline
\end{tabular}

Notes: * SDO - Scattered Disk Object; Ref: A - this paper; B - Matsuura et al. (1987); C Delsanti et al. (2004); D - Delsanti et al. (2006); E - McBride et al. (2003); F - Deressoundiram et al. (2003); G - Woodward et al. (1996).

suggests that the nucleus is rotating counter clockwise. Jets of gas and dust from comet nuclei are common features among comets. They come from active regions on the nucleus surface. Gas jets were observed in comets C/1996 B2 Hyakutake, C/1995 O1 Hale-Bopp, $109 \mathrm{P} /$ Swiff-Tuttle (found in long-slit spectra) and for the first time in comet $1 \mathrm{P} / \mathrm{Halley}$ (A'Hearn et al. (1986)). 

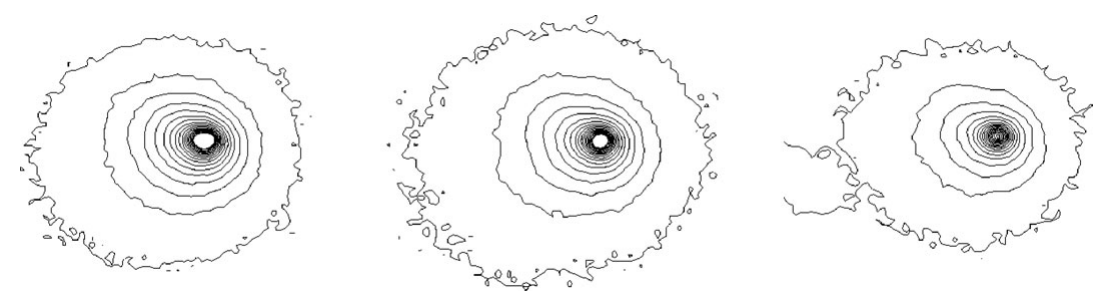

Figure 4. Isophotes of comet McNaught. From left to right, respectively, in J, H and K bands. The isophotes, in logarithmic scale, are asymmetric about the nucleus and show extension along anti-solar direction.
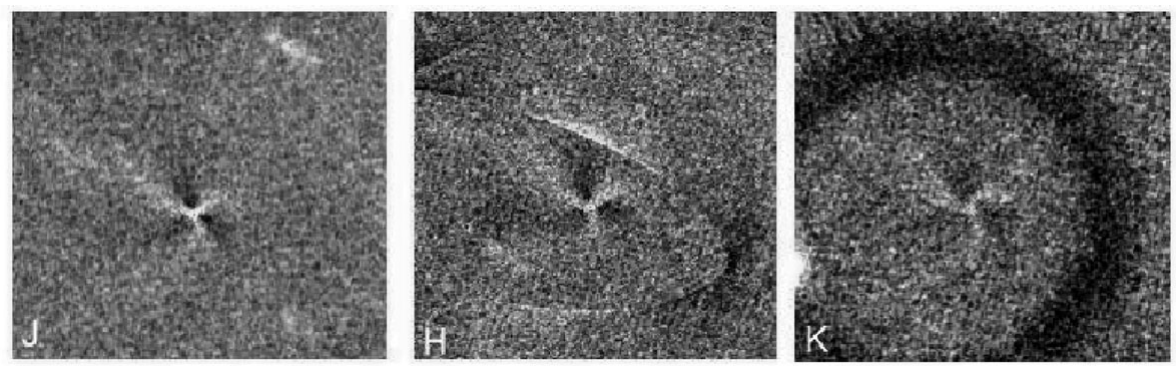

Figure 5. Morphology of comet McNaught; from left to right in $\mathrm{J}, \mathrm{H}$ and $\mathrm{K}$ bands. North is up and East is right. Field of view in arcsec: $\mathrm{J}(32 \times 29), \mathrm{H}(27 \times 28), \mathrm{K}(27 \times 28)$. Plate scale (in $\mathrm{km}$ ) is indicated.

\section{References}

A'Hearn, M. F., Hoban, S., Birch, P. V., Bowers, C., Martin, Ralph., \& Klinglesmith, D. A. 1986, Nature, 324, 649

Delsanti, A., Peixinho, N., Doressoundiram, A., Boehnhardt, H., Barucci, M. A., \& Large Program. 2004, Bulletin of the American Astronomical Society, 36, 1102

Delsanti, A., Peixinho, N., Boehnhardt, H., Barucci, A., Merlin, F., Doressoundiram, A., \& Davies, J.K. 2006, $A J, 131,1851$

Doressoundiram, A., Tozzi., G. P., Barucci, M. A., Boehnhardt, H., Fornasier, S., \& Romon, J. 2003, AJ, 125, 2721

Gehrz, R. D., Ney, E. P. 1992, Icarus, 100, 162

Matsuura, O. T., Picazzio, E., \& Kawara, K. 1987, PASP, 99, 154

McBride, N., Green, S. F., Davies, J. K., Tholen, D. J., Sheppard, S. S., Whiteley, R. J., \& Hillier, J. K. 2003, Icarus, 161, 501

Persson, S. E., Murphy, D. C., Krzeminski, W., Roth, M., \& Rieke, M. J. 1998, AJ, 116, 2475

Yoshida, S. 2008, http://www.aerith.net/comet/catalog/2005E2/2005E2.html, Updated on March 12 